\title{
Numerical analysis of choked converging nozzle flows with surface roughness and heat flux conditions
}

\author{
A ALPER OZALP \\ Mechanical Engineering Department, Uludag University, 16059, Bursa, Turkey \\ e-mail: aozalp@uludag.edu.tr
}

MS received 9 August 2004; revised 26 December 2005

\begin{abstract}
Choked converging nozzle flow and heat transfer characteristics are numerically investigated by means of a recent computational model that integrates the axisymmetric continuity, state, momentum and energy equations. To predict the combined effects of nozzle geometry, friction and heat transfer rates, analyses are conducted with sufficiently wide ranges of covergence half angle, surface roughness and heat flux conditions. Numerical findings show that inlet Mach and Nusselt numbers decrease up to $23.1 \%$ and $15.8 \%$ with surface heat flux and by $15.13 \%$ and $4.8 \%$ due to surface roughness. Considering each convergence half angle case individually results in a linear relation between nozzle discharge coefficients and exit Reynolds numbers with similar slopes. Heat flux implementation, by decreasing the shear stress values, lowers the risks due to wear hazards at upstream sections of flow walls; however the final $10 \%$ downstream nozzle portion is determined to be quite critical, where shear stress attains the highest magnitudes. Heat transfer rates are seen to increase in the streamwise direction up to 2.7 times; however high convergence half angles, heat flux and surface roughness conditions lower inlet Nusselt numbers by $70 \%, 15.8 \%$ and $4.8 \%$ respectively.
\end{abstract}

Keywords. Nozzle energy transport capability; surface wear hazard; discharge coefficient; transfer matrix.

\section{Introduction}

In a wide variety of engineering applications, nozzles are encountered in systems as the downstream components of complete running assemblies. A nozzle can be the flow accelerator in environmental control systems of commercial aircrafts (Vargas \& Bejan 2001), which supplies fresh air at certain temperature and pressure to the passenger cabin, it can also be the essential part of the exhaust system of nuclear propulsion engines (Kammash \& Godfroy 1997), which utilize fusion reactions to generate energy and thrust. Regardless of the objective, the accurate prediction in design-oriented calculations of compressible nozzle flows is still a challenging task for the aerodynamicist and achieves increasing importance when the nozzle performance is significantly influenced by geometry, inlet conditions and sources of nonisentropic character.

A list of symbols is given at the end of the paper. 
Nozzle performance was considered in many experimental and numerical studies, especially from the point of flow and heat transfer characteristics with various inlet-boundary conditions and flow geometries. Park et al (2001) investigated sonic nozzles that are applied to gas flow rate measurements and determined that the critical pressure ratio is highly dependent on the Reynolds number rather than area ratio especially in the cases with low flow velocity. Kim et al (2003) considered several kinds of gases and turbulence models with a wide range of Reynolds numbers on different sonic nozzle geometries. Instabilities in the propulsion of rockets, due to pressure and temperature fluctuations at the upstream of rocket nozzle and the flow geometry, were numerically considered by Assovskii \& Rashkovskii (2001). Variation of discharge coefficients for sonic nozzles with flow geometry and Reynolds number was reported by Paik et al (2000), who determined higher discharge coefficients with the increase of mass flow rate. Lear et al (1997) modelled dissipative effects of heat transfer on the exit kinetic energy and on nozzle efficiency and determined significant losses in efficiency due to heat transfer especially when the ratio of the inlet stagnation to back pressure converges to unity. Ahmad (2001) correlated the variation of nozzle discharge coefficient values for choked nozzle flows and Bartz (1957) handled the heat transfer phenomena in compressible nozzle flows and considered Nusselt number as a function of inlet stagnation pressure and convergence half angle. Massier et al (1970) conducted experiments in a $10^{\circ}$ convergence half angle nozzle with different working fluids and with a wide range of inlet stagnation pressures and recorded lower discharge coefficients with the decrease of inlet stagnation pressure. Sato et al (2000) presented the recent data of a real-time propulsion engine, which is equipped with the air-cooling system and Back et al (1996) performed an experimental investigation of flow and heat transfer in a choked nozzle with cooling.

The available literature deals either with the cooling or constant surface temperature investigations; however combined effects of convergence half angle, surface roughness and heating on compressible nozzle flows are not considered. The objective of the current work is to present the results of a new mathematical model, capable of performing comprehensive compressible choked converging nozzle flow analysis including the implementation of both surface roughness $(\varepsilon)$ and surface heat flux $(Q)$ conditions. Isentropic and non-isentropic computations are carried for choked converging nozzle systems (CCNS) for various convergence half angles $(\alpha), Q$ and $\varepsilon$ cases. The model is validated with previous experimental and numerical results and the combined effects of $\alpha, Q$ and $\varepsilon$ on the flow and heat transfer characteristics are discussed in detail.

\section{Numerical analysis}

\subsection{Modelling}

The overall aim is to build a predictive model for converging nozzle flows in the presence of surface roughness and constant heat flux conditions. The model should permit the determination of necessary design parameters such as nozzle geometry, boundary conditions for any given performance requirement depending on the applications, thus the main requirements are adaptability, simplicity and low calculation time. The calculations rely on the principles of mass and energy conservation and on the momentum and state equations applied to the control volume, given in figure 1. Nozzle outlines can be designated in various styles; the linearly narrowing form, that is taken under consideration in the present work, can be defined by (1a), which interprets the interaction of the main structural parameters of the nozzle, such 


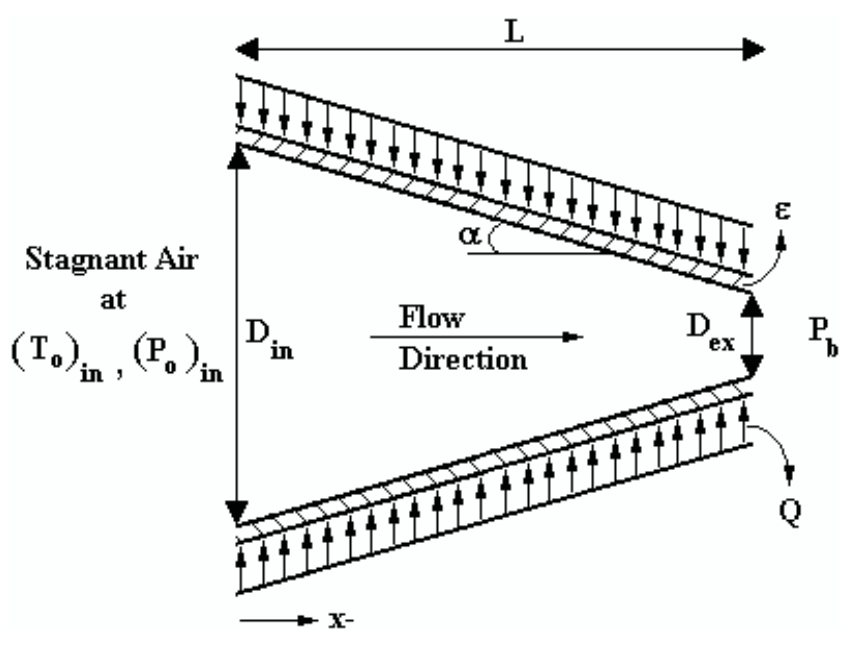

Figure 1. Nozzle outline.

as the inlet-exit diameters $\left(D_{\text {in }}-D_{\text {ex }}\right)$, length (L) and convergence half angle $(\alpha)$. Since the lateral and cross-sectional areas of the nozzle play a role in the complete set of the principal equations, streamwise variation of nozzle diameter appears to act influentially on the overall nozzle performance, and can be evaluated by rearranging (1a) in the form of (1b).

$$
\tan \alpha=\frac{D_{\text {in }}-D_{\text {ex }}}{2 L}, \quad D=D_{\text {in }}-2 x \tan \alpha
$$

The present model is based on the fact that the converging nozzle is operated steadily, thus the stagnation conditions of pressure and temperature, in the storage tank or turbine exit, upstream of the converging nozzle, are homogeneous and continuous. Moreover, as in many numerical works (Kammash \& Godfroy 1997; Lear et al 1997; Park et al 2001) the air velocity, pressure and temperature are considered to be uniform across any section normal to the flow axis. It is well known that air properties, like specific heat at constant pressure $\left(C_{p}\right)$, kinematic viscosity $(v)$ and Prandtl number (Pr), are substantially dependent on temperature (Incropera \& De Witt 1990); to sensitively implement the property variations with temperature into the calculations, the necessary air data of Incropera \& De Witt (1990) are fitted into $6^{\text {th }}$ order polynomials, which can be presented in closed form by (2a). The uncertainty of the fitted air data is less than $0.02 \%$ and the temperature dependency is indicated by the superscript $T$ throughout the formulation. To precisely assemble the interactions of the structural, compressible fluid, non-adiabatic \& frictional flow properties $(\xi)$ within the numerical model, the principal equations are reorganized to incorporate the nodal $\left(\xi_{i}\right)$ and the mean cellular property values $\left(\overline{\xi_{c}}\right)$, where the mean cellular values can be calculated by (2b).

$$
\xi^{T}=\sum_{j=0}^{6} a_{J} T^{J}, \quad \overline{\xi_{c}}=\frac{\xi_{c, i}+\xi_{c, i+1}}{2}
$$

Density $(\rho)$ variation, given by (3a), in the flow direction is one of the compressibility indicators; however as compressible flows are most likely to occur at elevated velocities, it is customary to define Mach number $(M)(3 b)$ as the ratio of the air velocity $(U)$ 
and speed of sound $(\sqrt{\gamma R T})$, where according to Laney (1998) the compressible character arises for $M>0 \cdot 3$. Mass flow rate $(\dot{m})$, being the most significant consideration from numerical point of view, is kept constant in the flow direction and can be calculated by $(3 \mathrm{c})$.

$$
\rho_{i}=\frac{P_{i}}{R T_{i}}, \quad M_{i}=\frac{U_{i}}{\sqrt{\gamma R T_{i}}}, \quad \dot{m}=\rho_{i} U_{i} A_{i}
$$

The friction coefficient $(f)$ is a function of both Reynolds number $\left(\mathrm{Re}_{D}\right)$ and surface roughness $(\varepsilon)(4 a)$, and Reynolds number (4b) is assigned to each differential cell with the mean cellular values of $U, D$ and $\nu$. On the other hand, the cell-based shear stress $(\tau)$ and friction force $\left(F_{f}\right)$ can be expressed with $(5 \mathrm{a}-\mathrm{b})$.

$$
\begin{aligned}
\frac{1}{\sqrt{\overline{f_{c}}}} & =-3.6 \log \left[\frac{6 \cdot 9}{\overline{\left(\operatorname{Re}_{D}\right)_{c}}}+\left(\frac{\varepsilon / \overline{D_{c}}}{3 \cdot 7}\right)^{1 \cdot 11}\right], \quad \overline{\left(\operatorname{Re}_{D}\right)_{c}}=\frac{\overline{U_{c} D_{c}}}{\overline{v_{c}^{T}}}, \\
\overline{\tau_{c}} & =\overline{f_{c}} \overline{\rho_{c}} \frac{\left(\overline{U_{c}}\right)^{2}}{2}, \quad \overline{\left(F_{f}\right)_{c}}=\overline{\tau_{c}} \pi \overline{D_{c}} \Delta x_{c} .
\end{aligned}
$$

One-dimensional momentum and energy equations (6) and (7) are applied to each differential cell in the nozzle, where the nodal properties such as $P, U$ and $C_{p}$ are interrelated with the contributions of cellular variants like $F_{f}, I, d q$ and $\Phi$. Equation (7) represents the conservation of mechanical and thermal energies by the implementation of cell based surface energy rate (8a) and the frictional loss term (8b), where the cellular lateral surface area $\left(\left(A_{s}\right)_{c}\right)$ can be evaluated by $(8 \mathrm{c})$.

$$
\begin{aligned}
P_{i} A_{i}+\dot{m} U_{i} & =P_{i+1} A_{i+1}+\dot{m} U_{i+1}+\overline{\left(F_{f}\right)_{c}}+\overline{I_{c}}, \\
\left(C_{P}^{T}\right)_{i} T_{i}+\frac{U_{i}^{2}}{2}+\overline{(d q)_{c}} & =\left(C_{P}^{T}\right)_{i+1} T_{i+1}+\frac{U_{i+1}^{2}}{2}+\overline{\Phi_{c}}, \\
\overline{(d q)_{c}} & =\frac{Q\left(A_{s}\right)_{c}}{\dot{m}}, \quad \overline{\Phi_{c}}=\frac{\overline{\left(F_{f}\right)_{c} U_{c}}}{\dot{m}}, \quad\left(A_{s}\right)_{c}=\pi \int_{x_{c, i}}^{x_{c, i+1}}\left(D_{i n}-2 x \tan \alpha\right) \mathrm{d} x .
\end{aligned}
$$

As work is involved in flows with friction and heat transfer, stagnation properties also vary in the flow direction, thus the conventional equations $(9 a-b)$ for compressible, isentropic and one-dimensional flows are applicable only with the simultaneous handling of the momentum and energy equations.

$$
\frac{\left(P_{o}\right)_{i}}{P_{i}}=\left(1+\frac{\gamma-1}{2} M_{i}^{2}\right)^{\frac{\gamma}{\gamma-1}}, \quad \frac{\left(T_{o}\right)_{i}}{T_{i}}=1+\frac{\gamma-1}{2} M_{i}^{2} .
$$

Vargas \& Bejan (2001) evaluated the heat transfer data in their mathematical model for a converging nozzle flow, where the Mach number was in the range of $0 \cdot 50-0 \cdot 85$, with the empirical correlation of (10a). In the current work, the Mach numbers are within 0.2-1.0, similar to the subsonic data of Vargas \& Bejan (2001), and (10a) is applied with the cell-based values of $f, \operatorname{Pr}$ and $\operatorname{Re}_{D}$. Moreover the combined effects of $\alpha, \varepsilon$ and $Q$ on $\dot{m}$ are investigated 
through the non-dimensional discharge coefficient $\left(C_{d}\right)$ of (10b), which compares the real mass flow rate with that of the isentropic case.

$$
\overline{\left(N u_{D}\right)_{c}}=\frac{\left(\bar{f}_{c} / 2\right)\left[\overline{\left(\operatorname{Re}_{D}\right)_{c}}-10^{3}\right] \overline{\operatorname{Pr}_{c}^{T}}}{1+12 \cdot 7\left(\overline{f_{c}} / 2\right)^{0.5}\left[\left(\overline{\operatorname{Pr}_{c}^{T}}\right)^{2 / 3}-1\right]}, \quad C_{d}=\frac{m_{\text {real }}}{m_{\text {isen }}} .
$$

\subsection{Computation}

For the one-dimensional, compressible marching procedure, forward difference discretization is applied in the flow direction, as defined by Chapra \& Canale (1990). Since the continuity, momentum and energy equations are to be solved in harmony, the geometric domain is divided into $n$ sequential cells, having equal widths of $\Delta x$. The fineness of the computational grids is examined to ensure that the obtained solutions are independent of the grid employed. Initial runs indicated that beyond 1000 cells the results showed no sign of change with grid density, thus to provide more reasonable predictions computations are performed with $n=1000$. Flow parameters, like $U, P, T, \rho$ and stagnation data $\left(P_{o}, T_{o}\right)$, are calculated at the nodes of these cells, that are numbered from $i=1$ to $n+1$, whereas $\tau, I, \operatorname{Re}_{D}$ and $\mathrm{Nu}_{D}$ are evaluated on cell basis using the mean values of nodal inlet and exit data of each cell.

As given in figure 2, by disregarding the surface roughness and heat transfer, the flow of the solution logic first handles the problem as of isentropic type, which is easy to manipulate as described by Laney (1998). The $M_{\text {in }}$ value of the isentropic approach is the initial guess of the iterative solution procedure of the non-isentropic nozzle flow. The non-isentropic approach governs the complete equation set described above, however for simultaneous handling they need to be assembled into the 3-dimensional "Transfer Matrix", which is the computational frame of the CCNS, consisting of the converted explicit forms of the principal equations.

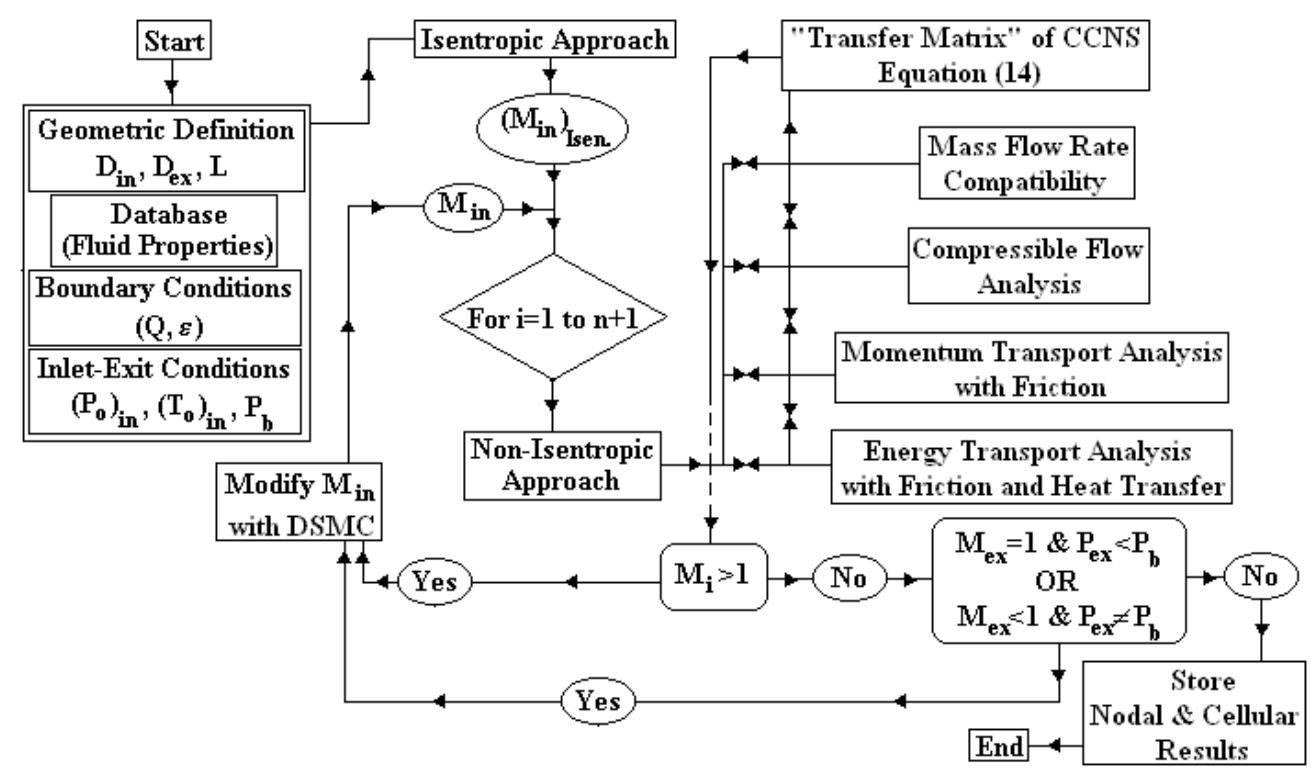

Figure 2. Flowchart of the numerical solution procedure for non-adiabatic and frictional compressible flow. 
Since air flow rate $(\dot{m})$ is constant in flow direction, by first substituting (3a) into (3c) and then equating the derivative of the new presentation $\left(\dot{m}=(\pi / 4 R)\left(P U D^{2} / T\right)\right)$ to zero forms a system of $n$ nonlinear equations, which completely represent the relation of geometric structure, static pressure, static temperature and velocity distributions (11). Moreover, defining the nodal impulse (I) and energy (E) levels in the forms of (12a) \& (12b) converts the momentum and energy equations into (13a) \& (13b), which identify the amount cellular momentum and energy transport rates. The proposed "Transfer Matrix" arrangement (14) is a 3-dimensional array of $3 \times 1000 \times 1000$, which is not only the numerical combination of the continuity, momentum and energy equations but also generates the computational domain of the nonadiabatic and frictional CCNS.

$$
\begin{aligned}
& \left(P_{i+1}-P_{i}\right)\left(\frac{\overline{U_{c}} \overline{D_{c}^{2}}}{\overline{T_{c}}}\right)+\left(U_{i+1}-U_{i}\right)\left(\frac{\overline{P_{c}} \overline{D_{c}^{2}}}{\overline{T_{c}}}\right) \\
& +\left(D_{i+1}-D_{i}\right)\left(\frac{\overline{P_{c}} 2 \overline{D_{c}}}{\overline{T_{c}}}\right)-\left(T_{i+1}-T_{i}\right)\left(\frac{\overline{P_{c} U_{c}} \overline{D_{c}^{2}}}{\overline{T_{c}^{2}}}\right)=0, \\
& I=P A+\dot{m} U, \quad E=\left(C_{P}^{T}\right) T+\frac{U^{2}}{2}, \\
& \Delta I=I_{i+1}-I_{i}=-\overline{I_{c}}-\overline{\left(F_{f}\right)_{c}}, \quad \Delta E=E_{i+1}-E_{i}=\overline{(d q)_{c}}-\overline{\Phi_{c}}, \\
& \Delta \text { transport }=\left[\begin{array}{c}
\Delta \dot{m} \\
\Delta I \\
\Delta E
\end{array}\right]_{i=1 \ldots n}=\left[\begin{array}{c}
\text { eq. }(11) \\
-I_{c}-\left(F_{f}\right)_{c} \\
(d q)_{c}-\Phi_{c}
\end{array}\right]_{i=1 \ldots n} \\
& =\left[\begin{array}{c}
0 \\
\left(P_{i+1} A_{i+1}-P_{i} A_{i}\right)+\dot{m}\left(U_{i+1}-U_{i}\right) \\
{\left[\left(C_{P}^{T}\right)_{i+1} T_{i+1}-\left(C_{P}^{T}\right)_{i} T_{i}\right]+\left(\frac{U_{i+1}^{2}}{2}-\frac{U_{i}^{2}}{2}\right)}
\end{array}\right]_{i=1 \ldots n}
\end{aligned}
$$

Although every single physical scenario is computationally incorporated by the 'Transfer Matrix' in the meshing domain, the sufficiently complex structure involves the highly dependent nonlinear formulations, where the solution scheme is most likely to face with convergence problems and encounter singularities, like $M_{i}>1, M_{e x}=1 \& P_{e x}<P_{b}$ or $M_{e x}<1 \& P_{e x} \neq P_{b}$. Direct Simulation Monte Carlo (DSMC) method, which was also applied by $\mathrm{Wu} \&$ Tseng (2001) to a gas dynamics problem, is recognized as a utilized technique especially for gas dynamics applications with instabilities and for internal compressible flow problems. An important feature of the present DSMC implementation is that the air motion and the influences of surface roughness and surface heat flux conditions are coupled over the meshing intervals of the flow domain; moreover cell-by-cell transport tracing technique is adopted both to the 'Transfer Matrix' scheme and to the DSMC algorithm, which offers greatest flexibility of handling different types of boundary conditions and requires much less specific change in programming. In order to perform accurate simulation for inlet/exit pressure boundaries and to sensitively evaluate the balance of heat swept from the nozzle walls and the energy transferred in the flow direction, the concept of triple transport conservation is also developed and incorporated into the DSMC. The basic idea is to update the inflow 
velocity by applying mass flux conservation, satisfying thermal equilibrium at each pressure boundary within the mesh, such that the mass flow rate conserves and the simulated boundary pressures coincide with the imposed values at the inlet and exit planes. The convergence criteria for the mass flow rate throughout the flow volume is of the order of $0.01 \%$ and successive non-isentropic runs are performed until the $M_{e x}$ is in the range of 0.99-1.0 and $P_{e x}$ is either above $P_{b}$ (underexpansion regime) or the shift of $P_{e x}$ from $P_{b}$ is less than $P_{b} \times 10^{-4}$ (design condition) (Laney (1998)).

\section{Results and discussion}

Numerical investigations are carried out with the nozzle convergence half angles of $\alpha=$ $0^{\circ}, 3^{\circ}, 6^{\circ}$ and $9^{\circ}$, with inlet stagnation to back pressure ratio $\left(\beta=\left(P_{o}\right)_{\text {in }} / P_{b}\right)$ of $\beta=2$, which corresponds to choking condition for the complete nozzle convergence half angle range. Influence of the surface roughness on the flow and heat transfer characteristics is simulated by applying 5 different non-dimensional cases of $\varepsilon / D_{\text {in }}=0.0025,0.0125,0.025,0.0375$ and 0.05 , moreover the effects of constant surface heat flux are evaluated by imposing 6 distinct values of $Q=20,400,800,1200,1600$ and $2000 \mathrm{~kW} / \mathrm{m}^{2}$. Results are discussed through streamwise variations of Mach number, shear stress, discharge coefficient and Nusselt number.

\subsection{Flow characteristics}

Variations in the streamwise $M$ are given in terms of $\alpha$ and $Q-\varepsilon$ in figures $3 \mathrm{a}-\mathrm{b}$ respectively. It can be seen from figure 3a that convergence half angle results in lower Mach numbers throughout the nozzle, except the exit value. When compared with the isentropic case of $\alpha=0^{\circ}, M_{\text {in }}$ values of the corresponding $\alpha=3^{\circ}, 6^{\circ}$ and $9^{\circ}$ flows are lower by $45.02 \%, 63.62 \%$ and $76.53 \%$ respectively. On the other hand the $M_{\text {in }}$ values of the highest $Q=2000 \mathrm{~kW} / \mathrm{m}^{2}$ case of $\alpha=3^{\circ}, 6^{\circ}$ and $9^{\circ}$ nozzles are lower than that of the $\alpha=0^{\circ}$ task by $34.74 \%, 56.39 \%$ and $72.01 \%$ respectively These proportions imply that $\alpha$ causes logarithmic decreases in $M_{i n}$; moreover the higher decrease rates in $M_{i n}$ due to convergence half angle put forward that $\alpha$ is more effective on $M_{i n}$ than surface heat flux. These findings are similar to those of Lear et al's (1997) numerical and Sato et al's (2000) experimental determinations. Application of the constant surface temperature condition on the nozzle wall (Lear et al 1997) caused flow velocities, thus $M$, to decrease, whereas the cooling of the nozzle surface (Sato et al 2000), which is the counter operation of the present work-focus, resulted in higher mass flow rates. For $\alpha=0^{\circ}$ case the heat flux of $Q=2000 \mathrm{~kW} / \mathrm{m}^{2}$ results in a decrease in $M_{i n}$ of $23.1 \%$ when compared with the isentropic flow. On the other hand the same heat flux constitutes lower $M_{\text {in }}$ by $8.72 \%, 7.84 \%$ and $7.62 \%$ for the $\alpha=3^{\circ}, 6^{\circ}$ and $9^{\circ}$ nozzles respectively. These proportions and figure $3 \mathrm{a}$ suggest that although the application of surface heat flux to high convergence half angle $\left(\alpha=6^{\circ}-9^{\circ}\right)$ nozzles, produces remarkable lower Mach numbers throughout the flow volume, the effect of heat flux on $M$ pattern is more apparent in cases with lower convergence half angles $\left(\alpha=0^{\circ}-3^{\circ}\right)$ The decrease of the Mach numbers, thus the mass flow rate values, due to the heat addition through the lateral nozzle walls is quite remarkable if the converging-nozzle is considered to be operated as a downstream component of a nuclear propulsion or jet/rocket engine, similar to those of Kammash \& Godfroy (1997) and Assovskii \& Rashkovskii (2001) respectively. The findings on Mach number variations through figure 3a imply that application of surface heat flux results in the heating process of the flowing air, causing augmented streamwise energy transport rates, where the thermal equilibrium of the nozzle energy transport capability can be balanced by lowering the mass flow rates. Indeed 
(a)

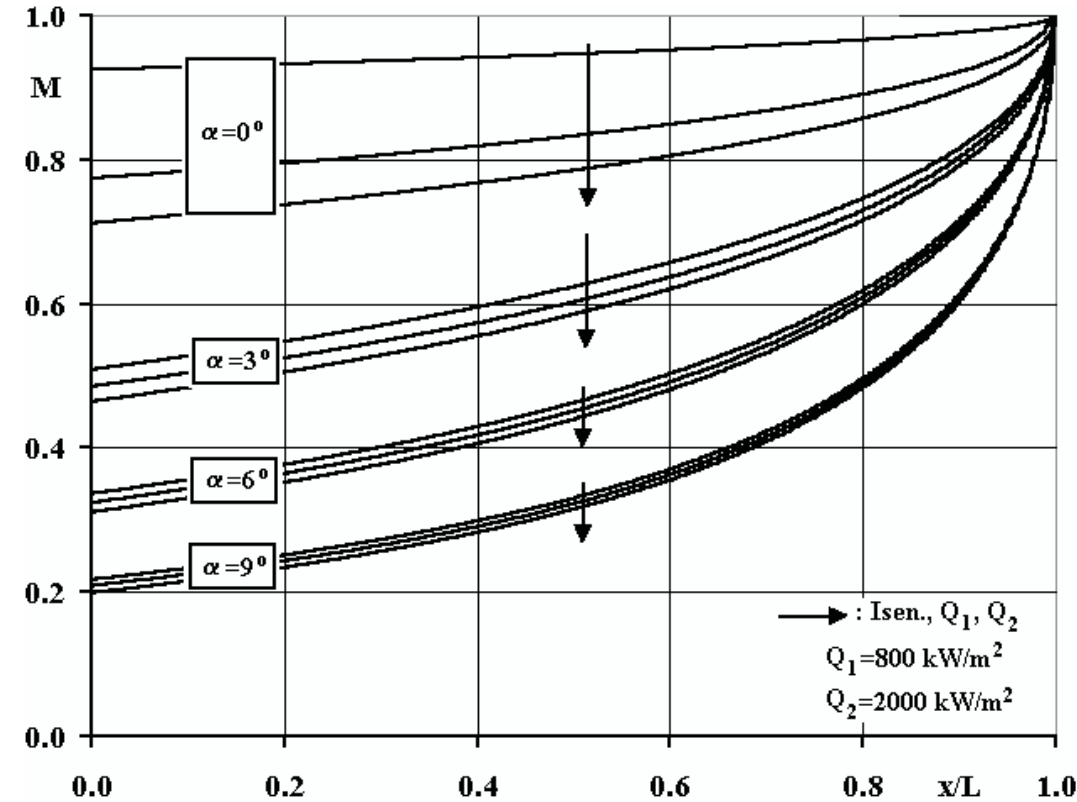

(b)

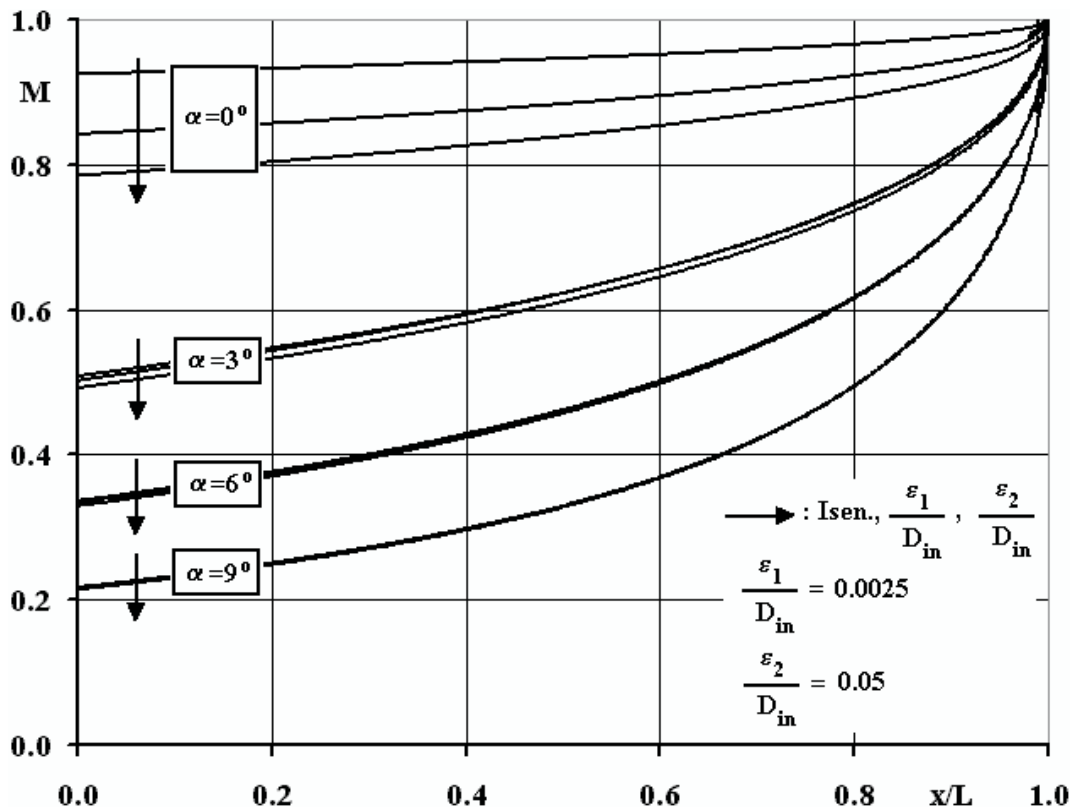

Figure 3. Influence of (a) heat flux and (b) surface roughness conditions on streamwise Mach number variations. 
this outcome is rather significant from the operation point of turbine, which operates at the upstream section and supplies air to the nozzle. Lower air flow rates cause decreased energy input to the nozzle at the inlet, which can either be achieved by running the turbine at lower rotational speeds or by lowering the fuel consumption rates in the combustion chamber of the propulsion or jet engine. Effects of various surface roughness cases on $M$ are compared with each other and with the isentropic flows in figure $3 b$. As in the heat flux discussions, surface roughness produces lower $M$ values, thus mass flow rates, for all $\alpha$ cases. The application of the non-dimensional surface roughness of $\varepsilon / D_{\text {in }}=0.0025$ to $\alpha=0^{\circ}, 3^{\circ}, 6^{\circ}, 9^{\circ}$ nozzles results in lower $M_{\text {in }}$ by $8.96 \%, 1.2 \%, 0.68 \%$ and $0.51 \%$ respectively, whereas else the corresponding decrease rates for $\varepsilon / D_{i n}=0.05$ are $15.13 \%, 3.25 \%, 2.11 \%$ and $1.38 \%$. It can be seen from figure $3 \mathrm{~b}$ that the influence of surface roughness on Mach number pattern is more apparent in nozzles with lower convergence half angles $\left(\alpha=0^{\circ}-3^{\circ}\right)$; however the effects decay in sharp converging cases $\left(\alpha=6^{\circ}-9^{\circ}\right)$ with the $M$ curves overlapping especially in the downstream sections. Nevertheless even the smallest decrease rates of the $\alpha=9^{\circ}$ case indicate that $\varepsilon$ itself, independent of the magnitude, is a resistive cause for compressible gas motion at high velocities of $0 \cdot 2<M<1 \cdot 0$. Moreover the divergence of the decrease rates, among the two $\varepsilon / D_{\text {in }}$ cases, becomes smaller as $\alpha$ is increased; which suggests that convergence half angle is more dominant than surface roughness, on Mach number pattern.

Shear stress variations, evaluated at constant $Q$ values of 20,800 and $2000 \mathrm{~kW} / \mathrm{m}^{2}$, with constant $\varepsilon / D_{\text {in }}=0.0125$ are presented in figure 4a. Higher $\alpha$ and $Q$ cause $\tau$ values to decrease, especially, at the upstream nozzle sections. Ribault \& Friedrich (1997) reported higher friction coefficients with surface cooling which is in harmony with the present findings. From the definition of shear stress (5a), the above defined reducing effects of higher $\alpha$ and $Q$ on $M$ and $\dot{m}$ values explain the lower values of $\tau$ beginning at the inlet section of the nozzles. Moreover towards downstream, the $\tau$ values of the higher $\alpha$ cases exceed those of the nozzles with smaller $\alpha$, which can be clarified by inspecting the variations of velocity, density and temperature values of the flowing air towards the $\tau$ intersection area. At the upstream neighbourhood of the intersection area, the difference of Mach numbers gets smaller (figure 3 ), $\rho$ and $T_{f}$ values of the higher $\alpha$ cases exceeds those of the smaller $\alpha$ by $10-18 \%$ and $10-70^{\circ} \mathrm{C}$ respectively. Increase of air viscosity with temperature and together with the augmentations in $M$ and $\rho$ data contributes the enhancement of sharp narrowing nozzle's $\tau$ data. Since shear stress is an indicator of surface wear, it can be said for the complete investigated nozzles that implementation of heat flux lowers the risks due to the wear hazards at the upstream sections of the flow walls; however the final $10 \%$ downstream $(0.9<x / L<1.0)$ portion is quite critical, where $\tau$ attains the highest values; it can additionally be implied that exit to inlet shear stress ratio $\left(\tau_{e x} / \tau_{i n}\right)$ increases sharply with higher $\alpha$ and $Q$. Figure $4 \mathrm{a}$ also indicates that, shear stress values of the cases with high $\alpha$ and low $M$ (figure 3) exceed those of the flows with low $\alpha$ and high $M$ in the 5\% neighbourhood $(0.95<x / L<1.0)$ of the nozzle exit, exposing the significance of the augmented wear data at the downstream nozzle sections. Figure $4 \mathrm{~b}$ presents $\varepsilon$ effects on $\tau$ distributions for various $\alpha$ cases. As expected, higher $\varepsilon / D_{\text {in }}$ resulted in higher $\tau$ values in the complete $\alpha$ set. On the other hand, $\tau_{i n}$ values, evaluated for $\varepsilon / D_{\text {in }}$ of 0.0025 and 0.025 , are separated by a factor of 2.06 for $\alpha=0^{\circ}$ and 2.09 for $\alpha=9^{\circ}$, whereas at the exit the ratios become 2.10 and 2.27 for $\alpha=0^{\circ}$ and $\alpha=9^{\circ}$ respectively. It is obvious that the proportions increase with both convergence half angle and streamwise direction, which is an outcome of the above described increase of density and flow temperature values in $\mathrm{x}$-direction and with higher $\alpha$. In addition, the exit to inlet $\tau$ ratios are 1.40 and 16.31 for the $\alpha=0^{\circ}$ and $\alpha=9^{\circ}$ nozzles respectively. These findings indicate that as $\alpha$ increases, the downstream nozzle walls are exposed to augmented local friction and flow temperature. 
(a)

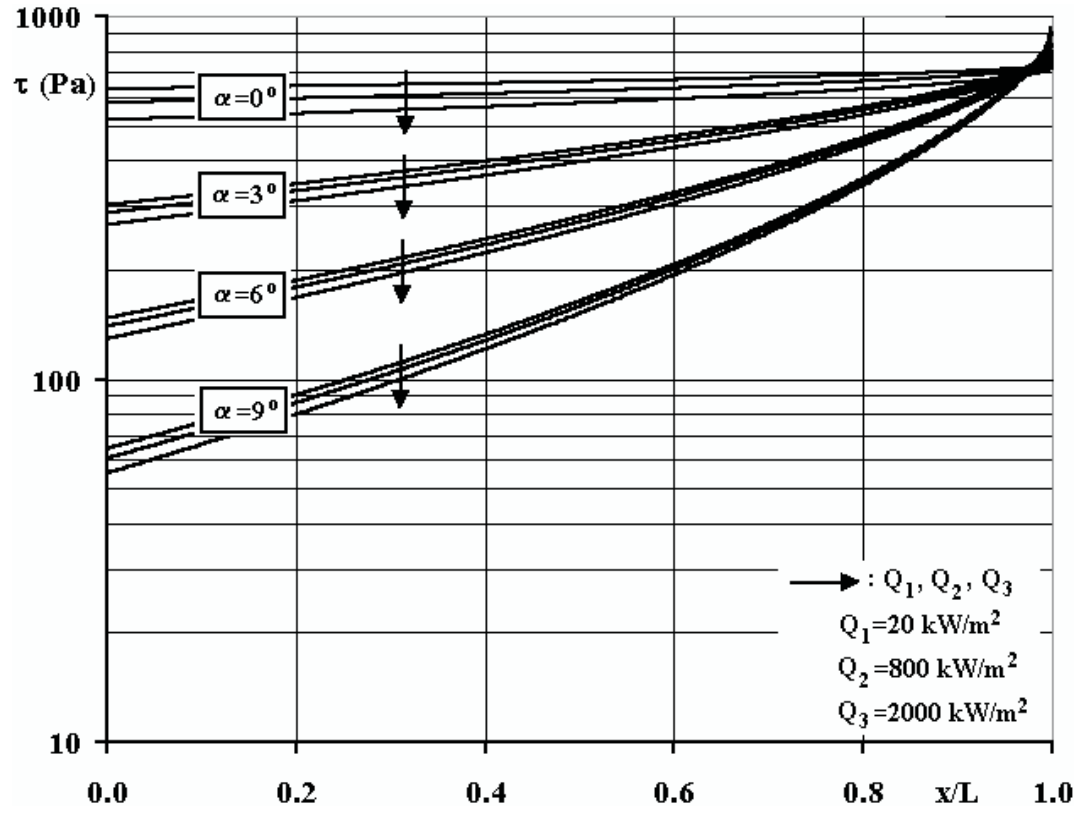

(b)

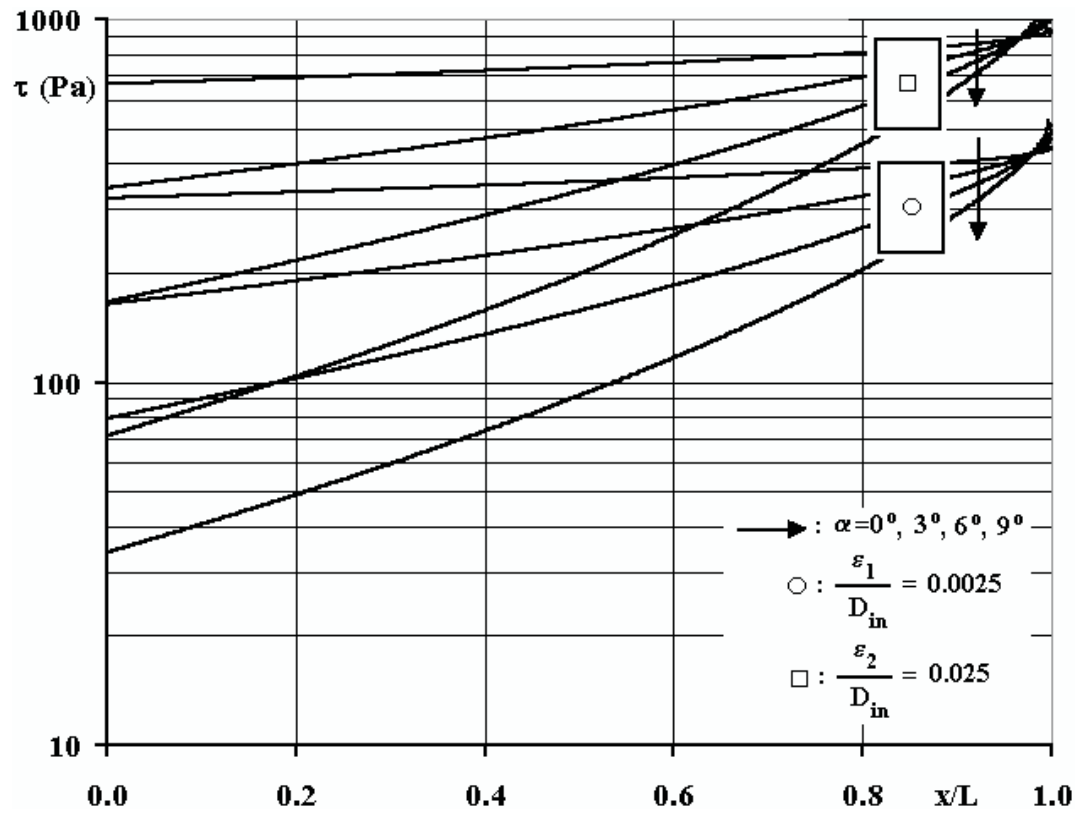

Figure 4. Influence of (a) heat flux and (b) surface roughness conditions on streamwise shear stress variations. 


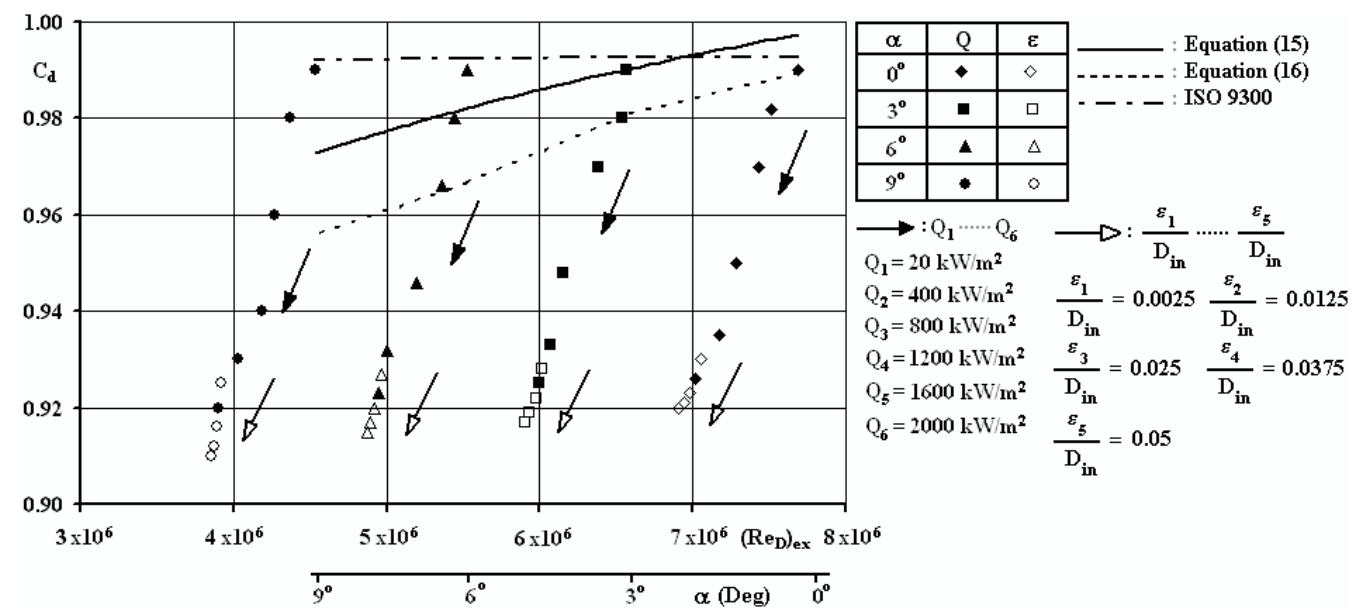

Figure 5. Influence of heat flux and surface roughness conditions on nozzle discharge coefficient variations.

Variation of discharge coefficient, in the presence of surface roughness and heat flux conditions is given in figure 5 with respect to nozzle convergence half angles and exit Reynolds numbers. The influence of heat flux is investigated by varying $Q$ in the range of $20-2000 \mathrm{~kW} / \mathrm{m}^{2}$ with the fixed $\varepsilon / D_{\text {in }}$ value of 0.0125 , whereas the surface roughness effects are evaluated through the applied $\varepsilon / D_{\text {in }}$ range of $0.0025-0.05$ by keeping $Q$ at $2000 \mathrm{~kW} / \mathrm{m}^{2}$. It can be seen from figure 5 that for the negligible $Q$ of $20 \mathrm{~kW} / \mathrm{m}^{2}, \mathrm{C}_{d}$ values converge to the $0.988-$ 0.992 interval, which is congenial with the experimental reports of Massier et al (1970) and Kim et al (2003), who ignored the heat transfer through nozzle walls in their investigations; moreover the interval is also in harmony with the ISO 9300 (Paik et al 2000) standardized correlation of $C_{d}=f\left(\left(\operatorname{Re}_{D}\right)_{e x}\right)$ for choked nozzles. Ahmad (2001) numerically evaluated the $C_{d}$ of various adiabatic nozzles and proposed (15-16), which characterize the influences of $\left(\operatorname{Re}_{D}\right)_{e x}$ and $\alpha$ on $C_{d}$ respectively. The highest deviation of the current $C_{d}$ values, for the cases with $Q=20 \mathrm{~kW} / \mathrm{m}^{2}$, from the correlations is $3.5 \%$ and figure 5 further implies that the gap decreases with lower $\alpha$ and with higher $\left(\operatorname{Re}_{D}\right)_{e x}$ values. The convergence of the $C_{d}$ values, under similar boundary and geometric conditions, to (15-16), also proves the validity of the demonstrated present computational model.

$$
\begin{aligned}
& C_{d}=0.2636+4.6279 \times 10^{-2} \ln \left(\operatorname{Re}_{D}\right)_{e x}, \\
& C_{d}=\left[1.0106+0.0074(\ln \alpha)^{2}\right]^{-1} .
\end{aligned}
$$

Although the nozzles are choked for the complete $\alpha$ range, higher convergence half angles produced lower exit $\operatorname{Re}_{D}$ as given in figure 5. The lower $\operatorname{Re}_{D}$ are accompanied with the reduced values of $C_{d}$ which put forward that the nozzles, with higher $\alpha$, keep the $M_{e x}$ of 1 with lower mass flow rates. The numerical results show, particularly for the $Q=2000 \mathrm{~kW} / \mathrm{m}^{2}$ case, that as $\alpha=0^{\circ}$ yielded $C_{d}$ of 0.927 the corresponding values for $\alpha=3^{\circ}, 6^{\circ}, 9^{\circ}$ are $0.925,0.923$ and 0.92 respectively, where Kim et al (2003) also reported lower $C_{d}$ with higher convergence half angles. Like $\alpha$, application of $Q$ decrease the $C_{d}$, which contributes to $M$ variations with $Q$ (figure 3a) and show parallelism with the reports of Paik et al (2000). If each convergence half angle case is considered individually, the decrease of $\left(\operatorname{Re}_{D}\right)_{e x}$ forms a linear relation with $C_{d}$ independent of the level of $\alpha$, with similar slopes. Figure 5 additionally 
shows that, at the lower and upper heat flux limits of $Q_{1}=20 \mathrm{~kW} / \mathrm{m}^{2}$ and $Q_{6}=2000 \mathrm{~kW} / \mathrm{m}^{2}$, the evaluated $\left(\operatorname{Re}_{D}\right)_{e x}$ for the convergence half angles of $\alpha=0^{\circ}, 3^{\circ}, 6^{\circ}$ and $9^{\circ}$ are in the intervals of $7.69 \times 10^{6} \rightarrow 7.02 \times 10^{6}\left(Q_{1} \rightarrow Q_{6}\right), 6.60 \times 10^{6} \rightarrow 5.99 \times 10^{6}, 5.52 \times 10^{6} \rightarrow$ $4.95 \times 10^{6}$ and $4.53 \times 10^{6} \rightarrow 3.90 \times 10^{6}$ respectively, where the ratio of the limits of these intervals $\left(\left[\left(\operatorname{Re}_{D}\right)_{e x}\right]_{Q 6} /\left[\left(\operatorname{Re}_{D}\right)_{e x}\right]_{Q 1}\right)$ result in the values of $0.913,0.908,0.896$ and 0.861 for $\alpha=0^{\circ}, 3^{\circ}, 6^{\circ}$ and $9^{\circ}$. The decrease of these ratios with $\alpha$, together with the linear relation of $\left(\operatorname{Re}_{D}\right)_{e x}$ with $C_{d}$, apparently indicate that the impact of heat flux on discharge coefficient grows with higher convergence half angles; this finding also coincides with the above determination on lower $C_{d}$ values with higher $\alpha$. The $C_{d}$ range of 0.99-0.92, due to heat flux application, is more significant than the above defined limits obtained for convergence half angle set, and clarifies that $Q$ is more effective on $C_{d}$ than $\alpha$. Like heat flux, implementation of higher surface roughness also decreases $\mathrm{C}_{d}$ in the complete $\alpha$ range. For the $\alpha=0^{\circ}$ case, the difference in $C_{d}$ among the $\varepsilon / D_{i n}=0.0025$ and $\varepsilon / D_{i n}=0.05$ cases is 0.01 , whereas the corresponding difference increases to 0.016 in the system with $\alpha=9^{\circ}$. As discussed in figure 3 , lower $M$ and $\dot{m}$ are the consequences of increased $\alpha$, thus these records indicate that the influence of $\varepsilon$ on $C_{d}$ increases in the systems having lower mass flow rates.

\subsection{Heat transfer characteristics}

Streamwise $\mathrm{Nu}_{D}$ variations are evaluated for different nozzle convergence half angle cases, by applying constant surface heat flux values of 20,800 and $2000 \mathrm{~kW} / \mathrm{m}^{2}$, with the constant value of $\varepsilon / D_{\text {in }}=0.0125$ and given in figure 6a. It can be seen that higher $\alpha$ and $Q$ result in lower $\mathrm{Nu}_{D}$, which can be attributed to the lower Mach number, air velocity and mass flow rate values in the narrower nozzles with higher heat flux values as discussed through figure $3 \mathrm{a}$. The augmentation in $\mathrm{Nu}_{D}$ towards downstream is an accompanied outcome of flow acceleration, and the ratio of the exit to inlet Nusselt numbers $\left(\lambda=\left(\mathrm{Nu}_{D}\right)_{e x} /\left(\mathrm{Nu}_{D}\right)_{i n}\right)$ increase with convergence half angles whereas decrease with heat flux. These findings agree well with those of Bartz (1957), who reported augmented $\lambda$ ratios with sharper $\alpha$, also with the $\lambda=3.9$ record of Ahmad (2001) for a nozzle with $\alpha=45^{\circ}$. However Back et al's (1996) $\lambda$ of 1.61 for $\alpha=15^{\circ}$ is lower than the current predictions, where the most significant ratio of $\lambda=2.70$ is evaluated for the convergence half angle of $9^{\circ}$. Application of different heat flux values caused the inlet $N u_{D}$ vary by $\pm 7.9 \%, \pm 3.2 \%, \pm 2.91 \%$ and $\pm 2.83 \%$ for the nozzles with $\alpha=0^{\circ}, 3^{\circ}, 6^{\circ}, 9^{\circ}$ respectively. These proportions imply that the effect of heat flux, at the inlet, on $\mathrm{Nu}_{D}$ becomes more significant on nozzles with lower convergence half angle cases, where the Mach numbers and mass flow rates are higher when compared with sharp converging nozzles. On the other hand, application of the surface heat flux has significant impact on the wall $\left(T_{w}\right)$ and flow $\left(T_{f}\right)$ temperature values, where $T_{w}$ is above $T_{f}$ for the complete $\alpha$ range and throughout the flow volume. Computations show that as the $T_{s}$ values decrease in the streamwise direction, the contrary is true for $T_{f}$ in all cases. The decrease in rates of $T_{s}$ become more significant towards the nozzle exit especially for $x / L>0.95$, where the highest flow acceleration (figure 3 ) and shear stress values (figure 4 ) are also determined for all nozzles. Higher $\alpha$ and $Q$, both indicating lower mass flow rates, constitute higher $T_{s}$ and $T_{f}$, especially at the nozzle inlet; as the durability of the nozzle material is directly related to the $T_{s}$, the numerical investigations indicate upstream nozzle sections for critical consideration. Effects of surface roughness on the streamwise $\mathrm{Nu}_{D}$ variations, for the heat flux $2000 \mathrm{~kW} / \mathrm{m}^{2}$, are investigated by the application of 3 different $\varepsilon / D_{\text {in }}$ values of $0.0025,0.025$ and 0.05 and shown in figure $6 \mathrm{~b}$. The resistive effect of surface roughness on flow decreases $\mathrm{Nu}_{D}$ for the complete convergence half angle set, however the impact is more significant in 
(a)

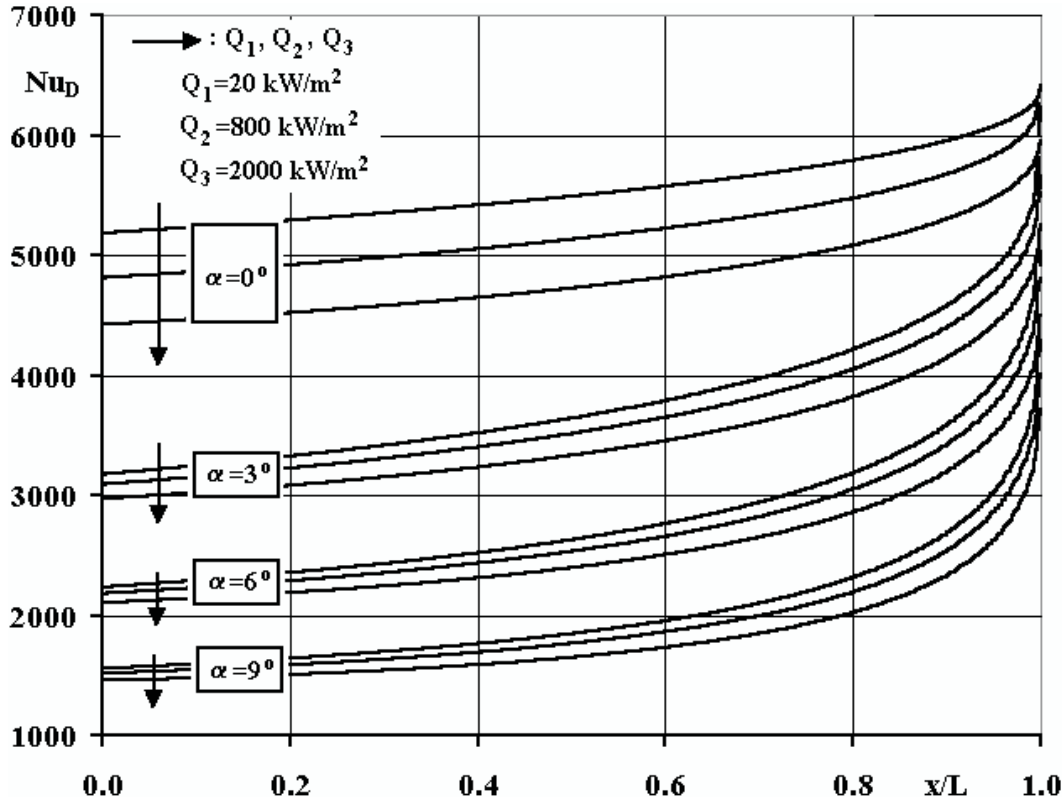

(b)

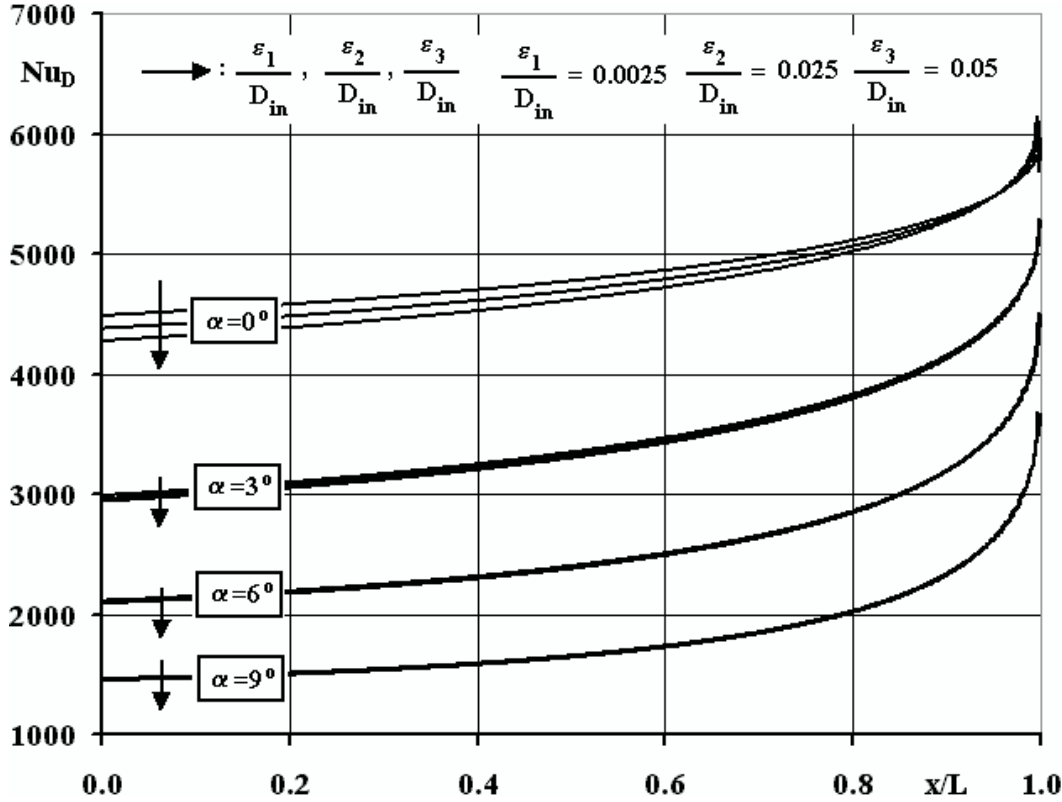

Figure 6. Influence of (a) heat flux and (b) surface roughness conditions on streamwise Nusselt number variations. 
the case with $\alpha$ of $0^{\circ}$, with a variation of $\pm 2.40 \%$ at the inlet. The gap among the $\alpha=0^{\circ}$ curves reduces towards downstream and at the exit $\mathrm{Nu}_{D}$ are within the range of $\pm 0.35 \%$; on the other hand as $\alpha$ is increased to $9^{\circ}$, the $\mathrm{Nu}_{D}$ curves for different $\varepsilon$ cases separate by less than $0.05 \%$. Figure 6 moreover implies that, when compared with that of the $\alpha=0^{\circ}$ case, inlet $N u_{D}$ decreases by $39 \%, 57 \%$ and $70 \%$ for $\alpha=3^{\circ}, 6^{\circ}$ and $9^{\circ}$ nozzles respectively, which indicates that surface heat transfer rates are influenced at most by convergence half angle and at least by surface roughness in choked converging nozzle flows.

\section{Conclusion}

Flow and heat transfer characteristics, in the presence of surface roughness and heat flux conditions, in choked converging nozzles, are investigated by means of a recently developed numerical model that integrates the developed "Transfer Matrix" of CCNS with the implemented DSMC method. Computations are carried for various convergence half angles and the model is validated with the available numerical and experimental data from the literature. It is determined that both surface roughness and heat flux conditions decrease mass flow rates and produce lower Mach and Nusselt numbers, however the effects of surface roughness on flow and heat transfer characteristics become negligible in cases with high nozzle convergence half angles. As heat flux causes decrease rates up to $23.1 \%$ in $M_{\text {in }}$ and $15.8 \%$ in $\mathrm{Nu}_{D}$, the corresponding values due to the application of surface roughness are $15.13 \%$ and $4.8 \%$ in $M_{i n}$ and $\mathrm{Nu}_{D}$ respectively; on the other hand convergence half angle plays the dominant role on $\mathrm{Nu}_{D}$ with the highest reduction amount of $70 \%$. In cases with lower mass flow rates, surface temperatures are recorded to increase significantly especially at the upstream nozzle sections, which is considerably notable from the point of nozzle material durability. Upstream values of the shear stress are found to decrease with higher convergence half angles and surface heat flux conditions however towards downstream the shear stress values of the higher convergence half angle cases exceed those of the nozzles with smaller convergence half angle. As heat flux causes a maximum decrease of $7 \%$ in discharge coefficient, the impact of heat flux on discharge coefficient is determined to increase with higher convergence half angles; moreover the effects of surface roughness and convergence half angle on discharge coefficient turn out to be similar with decrease in rates of $0.6 \%$ and $0.7 \%$ respectively.

\section{List of symbols}

a polynomial coeficient;

A cross sectional area, $\mathrm{m}^{2}$;

$C_{d} \quad$ discharge coefficient;

$C_{p} \quad$ constant pressure specific heat, $\mathrm{J} / \mathrm{kgK}$;

$D \quad$ nozzle diameter, $\mathrm{mm}$;

$d q \quad$ surface energy rate, $\mathrm{J} / \mathrm{kg}$;

$E \quad$ energy per unit mass, $\mathrm{J} / \mathrm{kg}$;

$f \quad$ friction coefficient;

$F_{f} \quad$ friction force, $\mathrm{N}$;

I thrust, $\mathrm{N}$;

$L \quad$ nozzle length, m; 


$\begin{array}{ll}\dot{m} & \text { mass flow rate, } \mathrm{kg} / \mathrm{s} ; \\ M & \text { Mach number; } \\ \mathrm{Nu}_{D} & \text { Nusselt number; } \\ P & \text { pressure, Pa; } \\ \mathrm{Pr} & \text { Prandtl number; } \\ Q & \text { surface heat flux, } \mathrm{W} / \mathrm{m}^{2} ; \\ R & \text { gas constant, } \mathrm{J} / \mathrm{kgK} ; \\ \mathrm{Re}_{D} & \text { Reynolds number; } \\ T & \text { temperature } \mathrm{K} ; \\ U & \text { air velocity, } \mathrm{m} / \mathrm{s} ; \\ x & \text { streamwise direction, } \mathrm{m} ; \\ \alpha & \text { convergence half angle, deg; } \\ \beta & \text { inlet stagnation to back pressure ratio; } \\ \varepsilon & \text { surface roughness, } \mathrm{mm} ; \\ \Phi & \text { frictional loss term, } \mathrm{m}^{2} / \mathrm{s}^{2} ; \\ \gamma & \text { specific heat ratio; } \\ \nu & \text { kinematic viscosity, } \mathrm{m}^{2} / \mathrm{s} ; \\ \rho & \text { density, } \mathrm{kg} / \mathrm{m}^{3} ; \\ \tau & \text { shear stress, Pa; } \\ \xi & \text { property. }\end{array}$

\section{Subscripts}

$\begin{array}{ll}b, o & \text { back, stagnation; } \\ c & \text { cell data; } \\ D & \text { diameter; } \\ \text { ex, in } & \text { exit, inlet; } \\ i, n & \text { node, cell number; } \\ s & \text { heat transfer surface. }\end{array}$

\section{Superscripts}

$T \quad$ temperature dependency;

- cellular average.

\section{References}

Ahmad R A 2001 Discharge coefficients and heat transfer for axisymmetric supersonic nozzles. Heat Transfer Eng. 22: 40-61

Assovskii I G, Rashkovskii S A 2001 Low-frequency instability of solid rocket motors: influence of the Mach effect and charge geometry. Combustion, Explosion Shock Waves 37: 321-330

Back L H, Massier P F, Cuffel R F 1996 Some observations on reduction of turbulent boundary-layer heat transfer in nozzle. AIAA J. 4: 2226-2229

Bartz D R 1957 A simple equation for rapid estimation of rocket nozzle convective heat transfer coefficients. Jet Propulsion 27: 49-51

Chapra S C, Canale R P 1990 Numerical methods for engineers 2nd edn (Singapore: McGraw Hill) 
Incropera F P, De Witt D P 1990 Fundamentals of heat and mass transfer 3rd edn (New York: John Wiley \& Sons)

Kammash T, Godfroy T 1997 An open cycle gas core fusion rocket for space exploration. Acta Astronaut. 41: 229-237

Kim H D, Kim J H, Park K A, Setoguchi T, Matsuo S 2003 Computational study of the gas flow through a critical nozzle. Proc. Instn. Mech. Engrs. 217: 1179-1189

Laney C B 1998 Computational gasdynamics (Cambridge: University Press)

Lear W E, Sherif S A, Langford J R 1997 Efficiency and gas dynamics analysis of two-phase mixtures in supersonic nozzles with inter-phase heat transfer and slip. Acta Astronaut. 40: 701-706

Massier P F, Back L H, Noel M B, Saheli F 1970 Viscous effects on the flow coefficient for supersonic nozzle. AIAA J. 8: 605-607

Paik J S, Park K A, Park J T 2000 Inter-laboratory comparison of sonic nozzles at KRISS. Flow Measure. Instrum., 11: 339-344

Park K A, Choi Y M, Choi H M, Cha T S, Yoon B H 2001 The evaluation of critical pressure ratios of sonic nozzles at low Reynolds numbers. Flow Measure. Instrum. 12: 37-41

Ribault C L, Friedrich R 1997 Investigation of transport equations for turbulent heat fluxes in compressible flows. Int. J. Heat Mass Transfer 40: 2721-2738

Sato T, Tanatusgu N, Naruo Y, Kashiwagi T, Omi J, Tomike J, Nishino T 2000 Development study on ATREX engine. Acta Astronaut. 47: 799-808

Vargas J V C, Bejan A 2001 Thermodynamic optimization of finned crossflow heat exchangers for aircraft environmental control systems. Int. J. Heat Fluid Flow 22: 657-665

Wu J S, Tseng K C 2001 Analysis of micro-scale gas flows with pressure boundaries using direct simulation Monte Carlo method. Comput. Fluids 30: 711-735 